\title{
High-Resolution Digital Teleradiology: A Perspective
}

\author{
Gopinath R. Kuduvalli, Rangaraj M. Rangayyan, and J.E. Leo Desautels
}

Teleradiology has come a long way, from analog transmission systems using slow-scan television over standard telephone lines, to present-day, commercially available, microcomputer-based, low-resolution teleradiology systems. However, there exists a need to address the high-resolution end of the medical imaging categories, namely chest radiographs and mammograms, to firmly establish teleradiology. The availability of high-resolution image digitizers, display units, and digital hard copiers has made high-resolution digital teleradiology a feasible concept. Although the use of satellite channels can speed up the transmission of radiographic image data, with widespread acceptance of high-resolution teleradiology systems in the foreseeable future, the sheer amount of data involved in this field will give rise to problems of data transmission and storage. Data compression schemes can bring down the amount of data handled and can have a great economic impact on future teleradiology systems. We have developed a number of compression techniques for reversible compression of medical images. Our experiments have shown that lossless compression of the order of $4: 1$ is possible for a class of high-resolution medical images. Use of pattern recognition techniques offers the potential to bring down these data rates even further. We plan to use these techniques in a prototype high-resolution teleradiology system being developed. In this paper, we trace some of the developments in teleradiology and image data compression, and present a perspective for teleradiology in the 1990s.

Copyright $\odot 1991$ by W.B. Saunders Company

KEY WORDS: teleradiology, image coding, data compression, image transmission.

$\mathbf{T}$ ELERADIOLOGY is defined as the practice of radiology at a distance. ${ }^{1,2}$ Teleradiology offers a technologic approach to the problem of eliminating the delay in securing consultation of a radiologist to patients in rural and remote areas. Timely availability of radiologic diagnoses via telecommunication could potentially reduce morbidity and mortality of people in remotely situated areas and in developing countries, and costs of transportation to tertiary health-care centers. In the military environment, where one study in the United States in $1983^{3}$ indicated that over $65 \%$ of the medical facilities with radiographic equipment have no radiologists assigned to them and an additional $15 \%$ have only one radiologist, teleradiology is an ideal vehicle for redistributing the imagereading work load from understaffed sites to more adequately staffed central locations. ${ }^{4}$ The province of Alberta, Canada, has 130 healthcare centers with radiologic imaging facilities; only 30 have resident radiologists. Sixty-one of the other centers depend on visiting radiologists. The remaining 39 centers send their radiographs to other centers for interpretation, with a 3- to 14-day delay in receiving the results. ${ }^{5}$ The situation is comparable in the neighboring provinces of Saskatchewan and Manitoba. These three provinces could benefit significantly from teleradiology. Even in the case of areas served by contract radiologists, teleradiology would permit evaluation and consultation by competent radiologists in tertiary health-care centers in emergency situations as well as in complicated cases.

Early attempts at teleradiology systems ${ }^{6,7}$ consisted of analog transmission of slow-scan television over existing telephone lines, ultra-high frequency (UHF) radiolinks, and other such analog channels. ${ }^{2}$ Analog transmission and the concomitant slow transmission rates were entirely satisfactory for low-resolution images, such as nuclear medicine images. However, the transmission times were prohibitively high for higher resolution images, such as chest radiographs. ${ }^{2}$ Further, the quality of images received with analog transmission is a function of distance, which results in an unpredictable performance of radiologists with the received images. Thus, the natural progression of teleradiology systems was toward digital image formats. The initial choice of a transmission medium was again the ordinary telephone line operating at 300 bits per second (bps) to $1,200 \mathrm{bps}$. Many of the commercially available teleradiology sys-

From the Department of Electrical and Computer Engineering, The University of Calgary, and the Department of Radiolog. ical Sciences and Diagnostic Imaging, Foothills Hospital, Calgary, Alberta, Canada.

Supported by grants from the Natural Sciences and Engineering Research Council of Canada.

Address reprint requests to Dr R.M. Rangayyan, Department of Electrical and Computer Engineering, The University of Calgary, Calgan, Alberta T2N IN4, Canada.

Copyright 11991 by W.B. Saunders Company

0897-1889/91/0404-0005\$03.00/0 
tems still use telephone lines for transmission. Recent improvements in modem technology, allowing speeds up to $19.2 \mathrm{Kbps}$ over standard telephone lines, and the establishment of a number of $56-\mathrm{Kbps}$ lines for commercial use by telephone companies, have made this medium a viable one for low-resolution images. ${ }^{8}$

A major reason for users' reluctance in accepting teleradiology systems has been the inability to meet the resolution of the original film image with the digitized and received images. This has been so, for example, for mammograms and chest radiographs. Even a spatial resolution of $2,048 \times 2,048$ cannot capture the submillimeter features on these images. ${ }^{9}$ Thus, a spatial resolution of 4,096 $\times 4,096$ pixels or better, and at least 1,024 shades of gray, are required to capture all the diagnostic information on these images. The recent development of highresolution digitizers capable of digitizing to a spatial resolution of $4,096 \times 4,096$ pixels with 4,096 shades of gray ( 12 bits) has alleviated this problem. But now, teleradiology system designers are faced with the problem of dealing with the immense amount of data involved in such high-resolution images. Transmission of such large amounts of data over ordinary telephone lines involves large delays, ${ }^{2}$ and can be overcome to some extent by using parallel dial-up lines for increased data transfer rate. Even if the use of satellite channels can speed up their transmission, ${ }^{10}$ the anticipated widespread acceptance of high-resolution teleradiology systems in the foreseeable future will create archival and image data management problems. This is the motivation for looking into data compression techniques. However, having gone a considerable length to obtain high-resolution images, any loss of information in compression and decompression which could result in possible degradation of performance of radiologists with those images and should be avoided.

Significant image data compression may be achieved by decorrelation of or reduction in redundancy in the image data. Redundancy in an image depends on the nature of the image and spatial resolution or scale of digitization, among other factors. Lossless compression of the order of $4: 1$ is possible on a class of high-resolution medical images, decreasing the transmission and storage requirements by the same factor. ${ }^{11}$ Further, medical images such as chest and skull radiographs at times have regions surrounding the central image that do not carry significant diagnostic information. In the past, binary masks and predefined boundaries at a certain scale of digitization have been used for eliminating such regions from coding. ${ }^{12,13}$ Moreover, in some medical images, only certain features are required for certain kinds of diagnosis: for example, in the case of chest radiographs, only the lung tissue might be of interest for certain types of diagnosis. In recent years, attempts have been made to model images using scene analysis techniques ${ }^{14,15}$ for data compression. They have been studied under the name analysis-synthesis methods. A combination of scene analysis techniques with decorrelation methods can be used for extracting only those regions of the image that are of interest, and to encode these regions with no loss of quality. An integrated image analysis and compression system would be at the heart of a future teleradiology terminal. In this paper, we trace some of these developments and present a perspective for teleradiology in the 1990s.

\section{HISTORICAL BACKGROUND}

The first instance of transmitting picture information for medical diagnosis dates back to 1950 when Gershon-Cohen and Cooley used existing telephone lines and a facsimile system adapted to convert medical images into video signals for transmitting images between two hospitals $45 \mathrm{~km}$ apart in Philadelphia. ${ }^{16}$ In a pioneering work in 1959, Jutras ${ }^{17}$ conducted what is perhaps the first teleradiology trial by interlinking two hospitals $8 \mathrm{~km}$ apart in Montreal with a coaxial cable link to transmit telefluoroscopy examinations. The potential of teleradiology for securing consultation of a radiologist in remotely situated areas, and redistribution of radiologists' load from understaffed centers to more adequately staffed centers, was immediately recognized, and a number of clinical evaluation projects got underway. ${ }^{2,6,7,18-23}$ Most of the early attempts consisted of analog transmission of medical images via standard telephone lines, dedicated coaxial cables, UHF radio, microwave, and satellite channels, and display on television monitors at the receiving terminal. James et al. ${ }^{23}$ give an excellent review of the 
results of the early experiments. Andrus and Bird $^{1}$ describe a conceptual teleradiology system in which the radiologist, stationed at a faraway medical center, remotely controls a video camera to zoom in on selected areas of interest and observes the results in real time on a television screen. Steckel ${ }^{24}$ conducted experiments with such a system using an 875-line, closed-circuit television system for transmitting radiographic images within a hospital for educational purposes, and concluded that the system's utility far outweighed disadvantages, such as the inability to view a sequence of images belonging to a single study.

In 1972, Webber and Corbus ${ }^{6}$ used existing telephone lines and slow-scan television for transmitting ordinary radiographs and nuclear medicine images. The resolution achieved was entirely satisfactory for nuclear medicine images, but both the spatial resolution and the gray scale dynamic range (radiometric resolution) were found to be grossly inadequate for ordinary radiographs. A somewhat similar experiment using telephone lines and slow-scan television by Jelasco et $\mathrm{al}^{7}$ resulted in $80 \%$ correct interpretation of radiographs. Other experiments with slow-scan television over telephone lines $^{23}$ demonstrated the inadequacy of this medium, and that the diagnostic accuracy in such a scheme was a function of the nature of the images being diagnosed.

Webber et $\mathrm{al}^{20}$ used UHF radiotransmission links in 1973 for transmitting nuclear medicine images and radiographs. Whereas the system worked satisfactorily for nuclear medicine images, evaluation of chest films needed zoom and contrast manipulation of the television monitor. Murphy et al $^{19}$ used a microwave link for transmission of images of chest radiographs acquired with a remotely controlled video camera, over a distance of about $4 \mathrm{~km}$, and concluded that this is an acceptable method for providing health care to those in remote areas.

Andrus et $\mathrm{al}^{18}$ transmitted images of abdomen, chest, bone, and skull radiographs over a $45-\mathrm{km}$ round loop, using a $4-\mathrm{MHz}, 512$-line television channel including three repeater stations. The television camera was remotely controlled using push buttons and a joystick to control zoom, aperture, focus, and pointing of the camera. They concluded that the television interpretations were of acceptable accuracy. Such real-time operation calls for special skills on the part of the radiologist, as well as coordination between the operator at the image acquisition site and the radiologist at the receiving center. Moreover, practical microwave links exist only between and within major cities, and cannot serve the communication needs of teleradiology terminals in rural and remote areas. Further, operating costs over the duration of interactive manipulations could be high and render such a scheme uneconomical.

In 1973, Lester et $\mathrm{al}^{21}$ used the ATS-1 satellite for analog transmission of videotaped radiologic information, and concluded that satisfactory radiographic transmission is clearly possible "if a satisfactory sensor of radiographic images were constructed." In 1979, Carey et $\mathrm{al}^{22}$ reported on the results of an analog teleradiology experiment using the Hermes spacecraft. They reported the effectiveness of television fluoroscopy to be $90 \%$ of that with conventional film. Page et $\mathrm{al}^{25}$ used a two-way analog television network using the Canadian satellite ANIK-B to transmit radiographic images from northern Quebec to Montreal, and reported an initial accuracy in television interpretations of $81 \%$ with respect to film reading. This value reached $94 \%$ after a 3-month training of the participant radiologists in the use of the television system. The noise associated with analog transmission, the low resolution of the thenexisting television monitors, and the requirement of radiologists to participate in real-time control of the image acquisition cameras made the concept of television transmission of radiographic images unacceptable. Further, the noise associated with analog transmission is distance dependent. Not surprisingly, James et $\mathrm{al}^{23}$ reported that their teleradiology system, transmitting emergency department radiographs via a satellite channel from a local television studio, was unacceptable due to the decrease in accuracy of image reading to $85 \%$ to $86 \%$ with respect to standard radiographs.

\section{DIGITAL TELERADIOLOGY AND THE STATE OF THE ART}

Given the many advantages of digital communication over analog methods, ${ }^{26}$ the natural progression of teleradiology was toward using 
digital techniques. The advent of a number of digital medical imaging modalities facilitated this trend. ${ }^{27,28}$ Digital imaging also allowed for image processing, enhancement, contrast scaling, and flexible manipulation of images on the display monitors after acquisition. Many of the initial attempts ${ }^{8,29-33}$ at digital teleradiology were based on microcomputers and used low-resolution digitization, display, and hard copiers. The resolution was on the order of $256 \times 256$ to $512 \times 512$ pixels with 256 shades of gray, mostly because of the nonavailability of high-resolution equipment. Gayler et $\mathrm{al}^{29}$ describe a laboratory evaluation of such a microcomputer-based teleradiology system. They used a $512 \times 512 \times$ 8 -bit pixel format for image acquisition and display, and evaluated radiologists' performance on routine radiographs. They found the performance to be significantly worse than that using film radiographs. Nevertheless, they concluded that microcomputer-based teleradiology systems "warrant further evaluation in a clinical environment."

In 1982, Rasmussen et $\mathrm{al}^{33}$ compared the performance of radiologists on images transmitted by analog and digital means with light-box viewing of films. The resolution of digitization used was $512 \times 256$, with 6 -bit pixels. The digital images were converted to analog signals for analog transmission. They concluded that the resolution used would provide satisfactory radiographic images for gross pathologic disorders, but would require higher resolution for subtle findings.

Gitlin et $\mathrm{al}^{31,32}$ followed their laboratory evaluation ${ }^{29}$ with a field trial using 9,600-baud standard telephone lines for transmission of $512 \times$ $512 \times 8$-bit images from five medical care facilities to a central hospital in Maryland. ${ }^{34}$ They reported ${ }^{32}$ a relative accuracy of $96.7 \%$ with video image readings compared with standard film interpretations-a substantially higher percentage than laboratory studies ${ }^{29}$ - and attributed the results to the large percentage of normal patients used in the sample, and to the higher clinical radiological experience of the video readers.

The 1984 field trial by Gitlin's group ${ }^{31}$ used a $1,024 \times 1,024$ matrix of pixels, 9,600-baud telephone lines, and lossy data compression to bring down the transmission times. They ob- served a relative accuracy of video readings of $87 \%$ with respect to standard film readings. The relative accuracy was clearly a function of the type of data compression used, among other factors.

Gordon et $\mathrm{al}^{35}$ present an analysis of a number of scenarios and tradeoffs for practical implementations of digital teleradiology. In related papers ${ }^{36,37}$ they further discuss the potential for providing advanced imaging services such as computed tomography through teleradiology.

In 1987, DiSantis et al ${ }^{38}$ digitized excretory urographs to $1,024 \times 1,024$-pixel matrices, transmitted them over standard telephone lines after data compression to a receiving unit approximately $3 \mathrm{~km}$ away, and used a panel of three radiologists to read the video images and compare them with the original film readings performed about a week earlier. Agreement of 93\% was found between film and video readings in the diagnosis of obstructions. However, only $64 \%$ of urethral calculi detected in the original radiographs were detected in the video images. This demonstrated clearly that whereas a resolution of $1,024 \times 1,024$ pixels is adequate for certain types of diagnosis, higher resolution is required for capturing all the diagnostic information on the original film.

In 1987, Kagetsu et $\mathrm{al}^{30}$ reported on the performance of a commercially available teleradiology system using $512 \times 512 \times 8$-bit images and transmission over 9,600-baud standard telephone lines after 2.5:1 compression. They conducted experiments with a wide variety of radiographs over a 4-month period, and reported an overall accuracy of $89 \%$ between film and video display. Based on these studies, they recommended a review of original films at some later date because of the superior spatial and contrast resolution of film.

A large number of present-day, commercial teleradiology systems are microcomputer-based, and use existing telephone lines for transmission of image data. Recent improvements in modem technology leading to $14.4 \mathrm{Kbps}$ and 19.2 Kbps transmission over standard telephones, and the installation of a number of 56 Kbps lines by telephone companies have improved this medium to some extent. The typical resolution of these low-resolution teleradiology 
systems is in the range of $256 \times 256$ to $512 \times$ $512 \times 8$-bit pixels. ${ }^{39-41}$ Some of the systems make use of lossy or lossless compression ${ }^{41,42}$ to meet data rate limitations of standard telephone lines. Some vendors are supplying image compression hardware for building into available teleradiology systems. ${ }^{43,44}$ Whereas such systems are adequate for handling the lower resolution of computed tomography, magnetic resonance, and nuclear medicine images, they are not suitable for handling the large format images such as chest radiographs and mammograms. Experiments with such systems have demonstrated the inadequacy of low-resolution digital teleradiology systems as an alternative to physical transportation of films/patients to centers with better radiological diagnostic facilities. Higher resolution is clearly called for and would result in larger amounts of data. Further, lossy data compression schemes to remain within the data rate limitation of telephone lines are clearly unacceptable.

The development of high-resolution image digitizers and display equipment, and routine utilization of high data-rate communication media, have paved the way for high-resolution digital teleradiology. In 1989, Carey et $\mathrm{al}^{10}$ reported on the performance of the commercially available DTR-2000 teleradiology system from DuPont, consisting of a $1,684 \times 2,048$ pixel laser digitizer with 4,096 quantization levels, a T1 satellite transmission channel (1.544 Mbps), and a DuPont laser film recorder with 256 possible shades of gray. DuPont uses a nonlinear mapping from the original 4,096 quantization levels to 256 levels on the film hard copy to make use of the fact that the eye is more sensitive to contrast variations at lower density. With this mapping, at the lower end of the gray scale, small differences in gray values correspond to larger differences in optical densities than at the higher end of the gray scale. Thus, the overall optical density of the film is much larger than can be obtained by linear mapping. Carey et $\mathrm{l}^{10}$ transmitted radiographic and ultrasonographic images over the system from Seaforth to London, Ontario, and reported an accuracy of $98 \%$ in reading laser-sensitive film. They concluded that the laser-sensitive film "clearly duplicated the original film findings." However, they also reported what they termed as contouring on the laser-sensitive film, which might have been due to the nonlinear mapping of the 4,096 original gray levels to 256 levels on the film. The problem with the nonlinear mapping is that certain portions of the original gray scale with rapidly changing gray levels are mapped into a single optical density on the film, giving rise to contouring artifacts.

Barnes et $\mathrm{al}^{8}$ suggested that the challenge of integrating the increasing number of medical imaging technologies can be met by networked multimodality imaging workstations. Cox et $\mathrm{al}^{45}$ made a comparison of images digitized to $2,048 \times 2,048 \times 12$ bits displayed on monitors with $2,560 \times 2,048 \times 8$-bit pixels, and digital laser film recording with conventional film. They reported significant differences in the performance of the three display formats: the digital hard copy performed as well as or better than conventional film, whereas the interactive display failed to match the performance of the other two. They suggested that although the differences could be eliminated by training the personnel in reading from displays, and by image enhancement techniques, it is premature to reach any conclusions.

A number of present-day, commercially available systems are approaching the resolution requirements of high-resolution digital teleradiology. The FilmFax teleradiology system from Discovery Systems Inc ${ }^{46}$ makes use of a laser film scanner with a resolution of $2,481 \times 2,048 \times$ 12-bit pixels and transmission over telephone lines to a receiving terminal with a laser film recorder and a high-resolution display monitor with 2,048 pixels/scanline. The resolution of the laser film recorder is not advertised. In addition to digitized films, the FilmFax teleradiology terminal can accept data from computed tomography, magnetic resonance imaging, and other digital medical imaging modalities.

An improved version of DuPont's DTR-2000 Digital Teleradiography System ${ }^{47}$ consists of a laser digitizer with a resolution of better than $4,000 \times 5,000 \times 12$-bit pixels, a laser film recorder with a spatial resolution of 200 lines/ $\mathrm{mm}$, and a customized look-up table for mapping the 12-bit digital image data to 256 shades of gray on the laser film. The transmission is at a maximum rate of $1.544 \mathrm{Mbps}$ over leased microwave links, coaxial cables, fiber-optic cables, or 
T1 satellite channels, depending on the distance. DuPont has also recently announced the availability of optical disks, alleviating the problem of image archival to some extent.

The Vortech Image Transmission Network from Vortech Data Inc ${ }^{48}$ uses DuPont's laser digitizer with a resolution of $1,684 \times 2,048 \times$ 12-bit pixels and satellite transmission at 1.544 Mbps. Their proprietary Medical Imaging Gateway (MIG) accepts the digital data, performs 2.5:1 lossless compression, transmits the data over a satellite channel, and is interfaceable to a number of standard digital medical imaging modalities. Optional ACR-NEMA interface ${ }^{49}$ is available for interfacing the MIG to other imaging systems.

Batnitzky et $\mathrm{a}^{50}$ have made an assessment of currently available technologies for film digitization, display, generation of hard copy, and data communication for application in teleradiology systems. They conclude that the currently available 2,048 $\times 2,048 \times 12$-bit laser digitizers, displays with 1,024 to 2,048, 8-bit to 12-bit pixels per scan line, hard copiers that interpolate $2,048 \times 2,048$-pixel matrices to $4,096 \times 4,096$ pixel matrices, and the merger of computer and communication technologies resulting in flexible wide-area networks, have paved the way for acceptance of "final interpretation teleradiology," completely eliminating the need to go back to the original films. Gillespy et $\mathbf{a l}^{51}$ described the installation of a DuPont Clinical Review System (CRS), consisting of a $1,680 \times 2,048 \times 12$-bit pixel laser digitizer, and a $1,024 \times 840 \times 12$-bit display unit, and report that "clinicians were generally satisfied with the unit." Studies of contrast and resolution of high-resolution digitizers ${ }^{52-54}$ have demonstrated that currently available digitizers are approaching the resolution of film.

Our present experimental set up at The University of Calgary consists of an Eikonix 1412 digitizing camera capable of digitizing films up to $4,096 \times 4,096 \times 12$ bits, a Megascan FDP-2111 frame buffer with a capacity of $4,096 \times 4,096 \times 12$ bits, and a Megascan UHR-2008 high-resolution display monitor with a $2,560 \times 2,048 \times 8$-bit display. Our experiments using a standard gray scale film strip indicated that after correcting for light intensity variations of the Gordon Instruments Plannar
1417 light box used to illuminate the films, and allowing for noise from all sources, the digitization can be considered useful up to 10 bits. ${ }^{5 S}$ With a T-1 satellite channel (1.544 Mbps digital channel, sometimes referred to as DS-1 channel to distinguish from the analog channels using PCM transmission with analog-to-digital and digital-to-analog conversion), transmission of a $4,096 \times 4,096 \times 10$-bit image takes about 2 minutes of the satellite channel time. ${ }^{56}$ The actual transmission times must address the 540-ms satellite response time, and will depend on the protocol used for communication. Tests are underway for determination of the turnaround times for transmission and reception of images using satellite channel simulators. The image data are formatted using the ACRNEMA message format. ${ }^{49,57,58}$

Clearly, with the advent of digitizers capable of 4,096 $\times 4,096$ pixels with 4,096 shades of gray, digital teleradiology is ready to serve as an alternative to transporting patients or films and for securing reliable diagnoses by radiologists to patients in remote areas. However, the large amount of data involved in high-resolution images has created archival and data transmission problems. Commercially available systems address the problem of transmission to some extent, but the archival of data would still remain a problem. Data compression can bring down the amount of data and have a significant impact on the future development of teleradiology and related technologies in the 1990s.

\section{IMAGE DATA COMPRESSION}

Recently, there has been considerable research activity in reversible compression of medical images. ${ }^{3,59-64}$ The requirement of high resolution in teleradiology resulting in enormous amounts of data, coupled with the fact that no loss of fidelity can be tolerated in compression and decompression in most medical applications, has motivated this interest. To capture all the diagnostic information in a chest radiograph or a mammogram, resolution of the order of $4,096 \times 4,096$ pixels with 10 to 12 bits/pixel is required. ${ }^{45}$ Transmission of such large amounts of data over ordinary telephone lines results in large delays. ${ }^{2}$ Although the use of high-data-rate satellite channels can alleviate the problem of transmission to some extent, ${ }^{10}$ 
with the anticipated widespread acceptance of high-resolution teleradiology systems in the foreseeable future, archival of the image data would still remain a problem. Data compression schemes can bring down the amount of data handled and can have a great economic impact on teleradiology systems.

Natural images have a high degree of redundancy due to the correlation of pixels with their surrounding pixels. Further, the pixel values are not uniformly distributed over the range of values possible. Information theory ${ }^{65-67}$ provides a basis for reducing redundancy, thereby achieving data compression. Accordingly, image data compression consists of two functions: decorrelation and encoding. Practical compression techniques combine decorrelation and coding in different ways. Further, in the case of some compression techniques, it may not be possible to perfectly reproduce the original image from the compressed one. Such compression techniques are said to be information lossy or irreversible. In contrast, the techniques in which the original image can be completely reproduced are said to be information preserving or reversible.

Image data compression has been investigated since the early $1970 \mathrm{~s}^{68-73}$ with various applications such as television, teleconferencing, and satellite imaging. With the advent of digital imaging modalities, interest in image compression in medical imaging gained momentum. $^{74}$ In most medical applications, no loss of information can be tolerated in compression and decompression of images. Some of the early studies involved direct application of existing image compression techniques and investigation of their suitability for medical images. Attempts have been made to quantify diagnostic information lost in compression and decompression. ${ }^{60}$ Techniques drawn from progressive image transmission, where a poor-quality image is first transmitted at very low data rates and the quality is progressively improved with additional transmission of data, have also been investigated $^{61,75-77}$ for reversible data compression. Recently, Roos et al ${ }^{59}$ made a comparison of various data compression techniques for medical images. They computed the entropy of images after application of a number of data compression techniques. However, they did not perform actual compression and decompression of images.

We have implemented a number of reversible image compression techniques for a proposed high-resolution digital teleradiology system. ${ }^{11,55,78,79}$ The techniques implemented are straight Huffman coding, ${ }^{80}$ Lempel-Ziv coding, ${ }^{81-83}$ twodimensional linear predictive coding, ${ }^{72,78,84}$ transform coding using the discrete Fourier-, discrete cosine-, and discrete Walsh transforms, ${ }^{85,86}$ linear interpolative coding, ${ }^{59}$ and combinations thereof. Compression and decompression using these techniques have been implemented and tested on some mammograms and chest radiographs digitized to about 4,000 $\times 4,000 \times 10$-bit pixels. ${ }^{11}$ We have achieved compression from 10 bits to 2.5 to 3.0 bits per pixel on these images without any loss of information. Some of these coding techniques, namely, linear predictive coding and transform coding, can result in higher compression when some errors in reconstruction are allowed. " Some of the techniques, namely transform coding and linear interpolative coding, can be adapted for progressive transmission of images. ${ }^{11,61,75-77}$ These are potential requirements in a high-resolution teleradiology system. A practical teleradiology terminal would have all these techniques at its disposal for requirement-specific application.

Medical images such as mammograms, and radiographs of the chest and head commonly have large regions surrounding the central image that do not carry significant diagnostic information. Some relatively simple techniques that require operator intervention for eliminating such regions from coding are the use of binary masks, ${ }^{12}$ and the use of predefined boundaries at a certain scale of digitization. ${ }^{13}$ Moreover, in some applications, diagnostic information may be confined to certain features of the image. For example, in the case of chest radiographs for detecting pulmonary nodules, only the lung tissue may be of interest; the ribs and pulmonary vessels constitute "subject noise" and do not carry significant diagnostic information..$^{27}$ Scene analysis techniques ${ }^{14,15,87}$ can be used to model such images for data compression. The idea of using pattern recognition methods in image coding is not new. As early as in 1971 , Berger ${ }^{67}$ pointed out that the field of rate distortion theory, in its broadest sense, 
encompassed the field of pattern recognition. However, practical coding methods based on scene analysis are relatively new. Forchheimer and Kronader ${ }^{14}$ gave an excellent review of the current state of the art in pattern recognitionbased image coding. One such method considers modeling the scene as a collection of threedimensional objects against a background, and coding each object and background region separately. The decoder synthesizes the objects from a priori descriptions. These techniques of coding are called analysis-synthesis methods. ${ }^{87}$ Such techniques have been used with a fair degree of success for modeling the three-dimensional structure of human faces by Platt and Badler, ${ }^{88}$ and by Parke. ${ }^{89}$ Welsh $^{90}$ used such a model for video telephone image compression, and coded the incremental changes in facial expressions and positions as changes in object orientation. Gray scale images can be modeled as random geometric models on a three-dimensional surface using fractals. ${ }^{91,92}$ Fractals have been used for scene analysis and coding of gross features of scenes, achieving large compression ratios. ${ }^{93-95}$ In the context of preserving the quality of medical images in encoding, scene analysis techniques can be used to automatically extract the regions of diagnostic interest and eliminate the background regions. Raghavan et $\mathrm{al}^{12}$ used binary masks for eliminating the background regions of magnetic resonance images. These masks were manually generated and had to be redefined for each image. Lo and Huang ${ }^{13}$ used predefined boundaries for eliminating regions of no diagnostic interest. These boundaries were defined for images taken by positioning the object at a particular viewing position of the digitizing camera. The concept of region/scene analysis for automatic elimination of the background in medical image coding is largely unexplored. We propose to look into region growing, ${ }^{96}$ scene analysis, ${ }^{97}$ and object modeling methods ${ }^{98}$ for extracting regions of diagnostic interest from medical images for subsequent coding using adaptive predictive coding techniques. We foresee that integrated image analysis and compression systems would be at the heart of future teleradiology terminals.

The introduction of compression and decompression in teleradiology systems raises the question of overall throughput of the system in transmission and reception, and in storage and retrieval of image data. In general, the available channel capacity is increasing at a much slower rate than the availability of high-speed computational hardware. Thus, it would be economically viable to incorporate necessary computational hardware at transmission/storage and reception/ retrieval ends for compression and decompression of images. However, compression of image data removes the inherent redundancy in images, thus making the data more sensitive to errors. ${ }^{99}$ In dedicated communication links, appropriate error control should be provided for detecting and correcting these errors. In the case of packet-switched communication links, removal of redundancy by data compression would result in increased retransmission overhead. However, with modern digital communication links operating at typical bit error rates of 1 in $10^{\circ}$, and channel utilization (throughput) efficiency of around $97 \%$ using modern highlevel, packet-switched protocols, ${ }^{100}$ the advantages of data compression far outweigh the overheads mentioned.

\section{DISCUSSION}

Teleradiology has come a long way from analog transmission systems using slow-scan television over standard telephone lines, to present-day, commercially available, microcomputer-based, low-resolution teleradiology systems. Some of the commercially available digital teleradiology systems are approaching the resolution requirements of high-resolution teleradiology as a feasible alternative to transportation of films/patients from remote areas to centers with better diagnostic facilities. The availability of high-resolution digitizers, display units, and digital hard copiers has made high-resolution digital teleradiology a feasible concept. However, the immense amount of data involved in the images will give rise to problems of transmission and storage. Data compression can bring down the amount of data involved in the transmission and storage of images generated at the remote sites and have a significant impact on the economics of the next generation of teleradiology systems. Commercially available systems have addressed the problem of transmission of 
data, but appear to have grossly ignored the potential of data compression techniques, optimized for medical images, to affect the economics of future teleradiology systems. We have developed a number of compression techniques for reversible compression of medical images. Our experiments with these compression techniques have shown that compression of the order of $4: 1$ is possible for a class of highresolution medical images. Use of pattern recognition techniques offers a significant potential to improve compression even further. We plan to use these techniques in the prototype teleradiology system being developed at The University of Calgary.

\section{ACKNOWLEDGMENT}

We thank Cory Royal and Claude Keon of Telesat Canada, Calgary, for their contributions to this work, and Dr Richard Gordon, University of Manitoba, Winnipeg, for introducing us to the subject of teleradiology and for his untiring efforts for the cause of teleradiology.

\section{REFERENCES}

1. Andrus WS, Bird KT: Teleradiology: evolution through bias to reality. Chest 62:655-657, 1972

2. Carey LS: Teleradiology: part of a comprehensive telehealth system. Radiol Clin North Am 23:357-362, 1985

3. Allman R: Potential contribution of teleradiology to the management of military. A Technical Report: Radiologist Resources in "Military Medicine," December 1983

4. Goeringer F, Mun SK, Kerlin BD: Digital medical imaging: implementation strategy for the defense medical establishment. Proc Society of Photo-optical Instrumentation Engineers, Medical Imaging III: PACS System Design and Evaluation 1093:429-437, 1989

5. Drew Consultants, Inc, Carlisle, MA: Market study for a high-performance teleradiology system, 1989

6. Webber MM, Corbus HF: Image communications by telephone. J Nucl Med 13:379-381, 1972

7. Jelasco DV, Southworth G, Purcell LH: Telephone transmission of radiographic images. Radiology 127:147149,1978

8. Barnes GT, Johnson GA, Staab EV: Teleradiology: Fundamental considerations and clinical applications. Presented at the 1989 RSNA Meeting, Chicago, IL. Unpublished lecture notes, 1989

9. Arenson RL, Sheshadri SB, Kundel HA, et al: Clinical evaluation of a medical image management system for chest images. AJR 150:55-59, 1988

10. Carey LS, O'Connor BD, Bach DB, et al: Digital teleradiology: Seaforth-London network, J Can Assoc Radiol 40:71-74, 1989

11. Kuduvalli GR, Rangayyan RM: Performance analysis of reversible image compression techniques for highresolution digital teleradiology. IEEE Trans Med Imaging (submitted)

12. Raghavan $\mathrm{S}$, Chatterjee $\mathrm{S}$, Waldron MB: Image compression applied to MRI images. Proc Int Conf IEEE Eng Med Biol Soc 11:526-527, 1988

13. Lo SBC, Huang HK: Error-free and irreversible radiographic image compression. Proc SPIE 536:170-177, 1985

14. Forchheimer R, Kronader T: Image coding-from waveforms to animation. IEEE Trans Acoust, Speech, Signal Processing ASSP-37;2008-2023, 1989
15. Geuen W: Principal strategy of model based source coding. Proceedings of the Picture Coding Symposium (PCS-83), June 1987, pp 165-166

16. Gershon-Cohen J, Cooley AG: Telegnosis. Radiology 55:582-587, 1950

17. Jutras A: Teleroentgen diagnosis by means of videotape recording. AJR 82:1099-1102, 1959 (editorial)

18. Andrus WS, Dreyfuss JR, Jaffer F, et al: Interpretation of roentgenograms via interactive television. Radiology 116:25-31, 1975

19. Murphy RL, Barber D, Broadhurst A, et al: Microwave transmission of chest roentegenograms. Am Rev Respir Dis 102:771-777, 1972

20. Webber MM, Wilk S, Pirrucello R, et al: Telecommunication of images in the practice of diagnostic radiology. Radiology 109:71-74, 1973

21. Lester RG, O'Foghludha F, Porter F, et al: Transmission of radiologic information by satellite. Radiology 109:731732,1973

22. Carey LS, Russell ES, Johnson EE, et al: Radiologic consultation to a remote Canadian hospital using Hermes spacecraft. J Can Assoc Radiol 30:12-20, 1979

23. James JJ, Grabowski W, Mangelsdorff AD: The transmission and interpretation of emergency department radiographs. Ann Emerg Med 11:404-408, 1982

24. Steckel RJ: Daily x-ray rounds in a large teaching hospital using high-resolution closed-circuit television. Radiology 116:25-31, 1975

25. Page G, Gregoire A, Galand C, et al: Teleradiology in Northern Quebec. Radiology 140:361-366, 1981

26. Kretz F, Nasse D: Digital television: transmission and coding. Proc IEEE 73:575-591, 1985

27. Kuni CC: Introduction to Computers and Digital Processing in Medical Imaging. Chicago, IL, Year Book Medical, 1988

28. Nudelman S: Historical perspectives on photoelectronic digital radiology, in James AE, Anderson JH, Higgins CB (eds): Digital Image Processing in Radiology. Baltimore, MD, Williams \& Wilkins, 1985, pp 1-27

29. Gayler BW, Gitlin JN, Rappaport W, et al: Teleradiology: An evaluation of a microcomputer-based system. Radiology 140:355-360, 1981 
30. Kagetsu NJ, Zulauf DRP, Ablow RC: Clinical trial digital teleradiology in the practice of emergency room radiology. Radiology 165:551-554, 1987

31. Gitlin JN: Teleradiology. Radiol Clin North Am 24:55-68, 1986

32. Curtis DJ, Gayler BW, Gitlin JN, et al: Teleradiology: Results of a field trial. Radiology 149:415-418, 1983

33. Rasmussen W, Stevens I, Gerber FH, et al: Teleradiology via the Naval Remote Medical Diagnosis System (RMDS). Proc SPIE, Picture Archiving and Communication Systems (PACS) for Medical Applications (Part I) 318:174-181, 1982

34. Skinner FL, Cerva J, Kerlin B, et al: The teleradiology field demonstration. Proc SPIE, Picture Archiving and Communication Systems for Medical Applications (Part I): 318:168-173, 1982

35. Gordon R, Rangayyan RM, Wardrop DH, et al: Improving image quality in teleradiology and tele-computed tomography. Proceedings of the IEEE International Conference on Systems, Man, and Cybernetics, New Delhi, India, 1983, pp 908-913

36. Rangayyan RM, Gordon R: Computed tomography from ordinary radiographs for teleradiology. Med Phys 10:687-690, 1983

37. Rangaraj MR, Gordon R: Computed tomography for remote areas via teleradiology. Proceedings of the SPIE, 1st International Conference on Picture Archival and Communication Systems (PACS) for Medical Applications, Newport Beach, CA, Vol 318 (Part I), January 18-21, 1982, pp 182-185

38. DiSantis DJ, Cramer MS, Scatarige JC: Excretory urography in the emergency department: utility of teleradiology. Radiology 164:363-364, 1987

39. Interrand Corp: The Medical Image Phone. Product literature. Chicago, IL, 1989

40. Image Data Corp: Teleradiology Product Family. Product literature. San Antonio, TX, 1989

41. Redi Vu systems: Tele-Vu 5000 Teleradiology Systems. Product literature. San Jose, CA, 1989

42. Advanced Video Products: TelePro-Teleradiology System. Product literature. Concord, MA, 1989

43. Telephoto Communications Inc: Alice Type- $120 \mathrm{im}$ age Compression System. Product literature. San Diego, CA, 1989

44. Optivision Inc: Optipac-Med: Medical Picture Archiving and Communications Module. Product literature. Davis, CA, 1989

45. Cox GG, Cook LT, McMillan JH, et al: Highresolution $2560 \times 2048 \times 12$ bit digital displays for chest radiography-A comparison with conventional film and digital hardcopy. Kansas City, KA, University of Kansas Medical Center, 1988

46. Discovery Systems Inc: FilmFax. Product literature. Denver, CO, 1989

47. DuPont Company: DTR-2000: Digital Teleradiography System. Product literature. Wilmington, DE, 1988

48. Vortech Data Inc: Vortech Image Transmission Network. Product literature. Richardson, TX, 1990

49. ACR-NEMA: ACR-NEMA digital imaging and communications standard 300-1988. Washington DC, National Electrical Manufacturers' Association, 1988
50. Batnitzky S, Rosenthal SJ, Siegel EL, et al: Teleradiology: an assessment. Radiology 177:11-17, 1990

51. Gillespy T, Staab EW, Lawrence E: Electronic imaging in a teaching hospital intensive care unit: evaluation of the clinical review system. J Digital Imaging 3:124-128, 1990

52. Lo SB, Gaskill JW, Mun SK, et al: Contrast information of digital imaging in laser film digitizer and display monitor. J Digital Imaging 3:119-123, 1990

53. Slasky BS, Gur D, Costa-Greco MA, et al: Receiver operating characteristic analysis of chest image interpretation with conventional, laser-printed, and high-resolution workstation images. Radiology 174:775-780, 1990

54. Yip K, Lubinsky, AR, Whiting BR, et al: Performance analysis of medical $\mathrm{x}$-ray film digitizers. Proc. SPIE, Medical Imaging IV: Image Formation 1231:508-525, 1990

55. Kuduvalli GR, Rangayyan RM: Reversible image compression techniques for high-resolution digital teleradiology. IEEE WESCANEX '90, IEEE Western Canada Conference and Exhibition on Telecommunications for Health Care: Telemetry, Teleradiology, and Telemedicine, Vol. 1355, July 6-7, 1990, pp 173-178

56. Keon C: Satellite communications for telemedicine: viability and economics. Presented at IEEE WESCANEX '90, IEEE Western Canada Conference and Exhibition on Health Care: Telemetry, Teleradiology, and Telemedicine, July 6-7, 1990

57. ACR-NEMA: ACR-NEMA data compression standard PS 2-1989. Washington DC, National Electrical Manufacturers' Association, 1989

58. Horii SC: The ACR-NEMA standards and their implications for teleradiology. IEEE WESCANEX '90, IEEE Western Canada Conference and Exhibition on Health Care: Telemetry, Teleradiology, and Telemedicine, Vol 1355, July 6-7, 1990, pp 152-163

59. Roos P, Viergiever MA, VanDijke MCA, et al: Reversible intraframe compression of medical images. IEEE Trans Med Imaging 7:328-336, 1988

60. Huang HK, Lo SBC, Ho BK, et al: Radiological image compression using error-free and irreversible, and two-dimensional discrete-cosine-transform coding techniques, J Opt Soc Am 4:984-992, 1987

61. Wang L, Goldberg M: Comparative performance of pyramid data structures for progressive transmission of medical imagery. SPIE Medical Imaging IV: Image Capture and Display 1232:403-413, 1990

62. Blume H, Fano A: Reversible and irreversible image data compression using the S-transform and Lempel-Ziv coding. Proc SPIE, Medical Imaging III 1091:2-18, 1989

63. Abramson JR, Krasner BH: Data compression in the PACS environment. Proc SPIE, Medical Imaging III 1091: 42-49, 1989

64. Bramble JH: Comparison of information-preserving and information-losing data-compression algorithms for CT images. Radiology 170:453-455, 1989

65. Shannon CE: A mathematical theory of communication. Bell Syst Tech J 27:379-423, 1948

66. Shannon CE: A mathematical theory of communication. Bell Syst Tech J 27:623-656, 1948

67. Berger T: Rate distortion theory. Englewood Cliffs, NJ, Prentice Hall, 1971 
68. Davisson LD, Gray RM (eds): Data compression (benchmark papers in electrical engineering and computer science). Stroudsberg, PA, Dowden Hutchinson \& Ross, 1976

69. Jain AK: Image data compression: a review. Proc IEEE 69:349-389, 1981

70. Netravali AN, Limb JO: Picture coding: a review. Proc IEEE 68:366-406, 1980

71. Mussman HG, Pirsh P, Grallert H: Advances in picture coding. Proc IEEE 73:523-548, 1985

72. Jayant NS, Noll P: Digital Coding of Waveforms. Englewood Cliffs, NJ, Prentice Hall, 1984

73. Netravali AN, Prasada B (eds): Visual Communication Systems. New York, IEEE Press, 1989

74. Todd-Pokropek A: Image data compression: A survey. Math Computer Sci Med Imaging 39:167-195, 1988

75. Wang L, Goldberg M: Progressive image transmission by transform coefficient residual error quantization. IEEE Trans Acoust, Speech, and Signal Proc ASSP-36:7576,1988

76. Elnahas SE, Tzou KT, Cox JR, et al: Progressive coding and transmission of digital diagnostic images. IEEE Trans Med Imaging MI-5:73-83, 1986

77. Cox JR, Moore SM, Blaine GJ, et al: Optimization of trade-offs in error-free image transmission. Proc SPIE, Medical Imaging III: Image Capture and Display 1091:1930, 1989

78. Kuduvalli GR, Rangayyan RM: Reversible compression of images by two-dimensional linear prediction. IEEE Trans Signal Processing (submitted)

79. Kuduvalli GR, Rangayyan RM: Linear predictive coding for reversible compression of medical images. Electronic Imaging West-90, Pasadena, CA, Vol. 2, 26 February-1 March, 1990, pp 912-916

80. Huffman DA: A method for the construction of minimum-redundancy codes. Proc Institute of Radio Engineers 40:1098-1101, 1952

81. Ziv J, Lempel A: A universal algorithm for sequential data compression. IEEE Trans Inform Theory IT-23:337343, 1977

82. Ziv J, Lempel A: Compression of individual sequences via variable-rate coding. IEEE Trans Inform Theory IT-24:530-536, 1978

83. Welch TA: A technique for high-performance data compression. Computer 17:8-19, 1984

84. Maragos PA, Schafer RW, Mersereau RM: Twodimensional linear prediction and its application to adap- tive predictive coding of images. IEEE Trans Acoust, Speech, and Signal Processing ASSP-32:1213-1229, 1984

85. Andrews HC, Pratt WK: Transform image coding, in: Proceedings of Computer Processing Communications. New York, NY, Polytechnic Press, 1969, pp 63-84

86. Habibi A, Wintz PA: Image coding by linear transformation and block quantization. IEEE Trans Commun Tech COM-19:50-63, 1971

87. Tian-Hu Yu, Mitra SK: A simple image anlysis/ synthesis technique and its application to image coding. Proc SPIE, Image Processing Algorithms and Techniques 1244:161-170, 1990

88. Platt SM, Badler NI: Animating facial expressions. IEEE Comput Graphics 13:245-252, 1981

89. Parke FI: Parameterised models for facial animation. IEEE Comput Graphics 12:121-133, 1982

90. Welsh WJ: Model based coding of video telephone images using an analysis/synthesis method. Proceedings of the Picture Coding Symposium (PCS-88), September 1988, paper 4.5

91. Mandelbrot B: Fractal Geometry of Nature. San Fransisco, CA, Freeman, 1982

92. Levy-Vehe J, Berroir J: 3-D fractal model for lung morphogenesis. Proc. SPIE, Medical Imaging IV: Image Processing 1233:20-22, 1990

93. Barnsley MF, Jacquin A, Malassenet F, et al: Harnessing chaos for image synthesis. ACM Computer Graphics 22:131-140, 1988

94. Wallach E, Karnin E: A fractal based approach to image compression. Proc ICASSP-86, Intl Conf Acoust, Speech, and Signal Processing 1:529-532, 1986

95. Demko S, Hodges L, Naylor B: Construction of fractal objects with iterated function systems. Computer Graphics 19:271-278, 1985

96. Haralick RM, Shapiro LG: Image segmentation techniques. Computer Vision, Graphics, and Image Processing 29:100-132, 1985

97. Duda RO, Hart PE: Pattern classification and scene analysis. New York, NY, Wiley, 1973

98. Shemlon S, Dunn SM: Rule-based image interpretation with models of expected structure. Proc SPIE, Medical Imaging IV: Image Processing 1233:33-44, 1990

99. Proakis JG: Digital communications. New York, NY, McGraw-Hill, 1989

100. Telesat Canada: Satellite Delay and Response Times. Product literature. Gloucester, Ontario, 1990 